\title{
Study on the Influence of Community Opening on Peripheral Road
}

\author{
Zhihang Zhou
}

\author{
School of Electrical \& Electronic Engineering, North China Electric Power University Baoding, Baoding \\ 071000, China \\ zhihangzhou@163.com
}

Keywords: factor analysis, graph theory, minimum cost, maximum flow.

\begin{abstract}
In this paper, the influence of residential opening on surrounding road traffic is studied. By using the minimum cost maximum flow problem in graph theory, the traveling vehicle is regarded as continuous flow; the maximum flow is used to characterize the carrying capacity of the traffic flow around the district; and the travel time is the cost. The key indexes influencing the road traffic before and after opening are calculated and analyzed by the Ford-Fulkerson algorithm and weighted adjacency matrix method respectively, and the different types of cells are discussed further.
\end{abstract}

\section{Introduction}

February 21, 2016, the State Council issued the views that a closed residential area is no longer built and the residential areas which have been completed are compound to gradually open. But can the open area improve road capacity, traffic conditions and optimize the road network structure?

Gated communities destroy the urban road network structure and block the city "capillaries". Therefore, it is likely to cause traffic congestion. After the opening, the road network density and road area increase, so the capacity will naturally improve. At the same time, although the increase in road access, the number of vehicles on the main road around the district and intersections of the district will increase. It may also affect the main road traffic.

\section{Measure index}

Urban road traffic system consists of a large number of roads and intersections formed by interweaving and the existing indicators are not systematic enough. In order to evaluate the road traffic systematically, the representative indicators are selected from three levels, namely, point (a crossing), line (a section of road) and surface (a regional transport networks) respectively.

There are some evaluation indexes that can be chosen, such as intersection delay time, speed satisfaction, traffic volume, saturation, travel time, speed intersection, road network density and so on ${ }^{[1]}$. But these indicators are more or less obviously connected, so the factor analysis ${ }^{[2]}$ can be used to explore and find that these indicators can eventually be turned into traffic maximum flow, the average time of two factors, which help solve the follow-up questions.

\section{Minimum time maximum flow vehicle traffic model}

In section 2, two key indexes (maximum traffic flow and average travel time) are evaluated by factor analysis. The opening of the community will introduce new roads, so as to ease the traffic pressure on the surrounding roads. However, as the number of intersections increases, the traveling speed of the vehicle is limited and the burden on the traffic network is increased. Therefore, the focus of the problem is whether the opening of the community can ensure the maximum traffic volume while reducing the average travel time of the traveler.

In order to facilitate the discussion, a typical intersection is taken as an example in this paper, as is shown in Figure 1. There are 20 major roads and 12 intersections. 


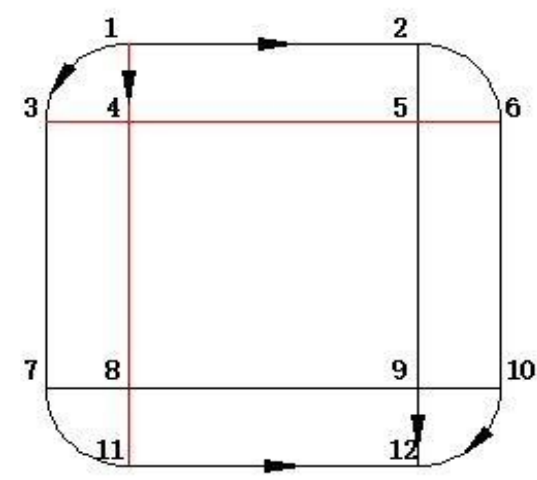

Fig. 1 Community road map

According to the principle of graph theory, the road intersection in Fig.1 is abstracted as the point in the graph. The road is abstracted as a line, denoted by $\mathrm{D}=(\mathrm{A}, \mathrm{E})$, where $\mathrm{A}$ is the vertex set with the intersection point as the element and $\mathrm{E}$ is the edge set with each road segment as the element.

The beginning and end of the flow path connected with the direction of its path has been identified, and other paths are possible to go in both directions. In addition to the arc connected to the beginning and end, the other arcs are directed. Now consider only from the A1 to A12 traffic flow.

Select $A_{1}$ as the starting point, denoted as $A_{s} ; A_{12}$ for the collection point, recorded as $A_{t}$, and the rest of the points are middle points. For each edge $\left(A_{i}, A_{j}\right) \in E$, there is a corresponding need to meet: $\mathrm{c}\left(A_{i}, A_{j}\right) \geq 0$. The capacity called edge is denoted by $c_{i j}$, which means the maximum traffic volume of the road. Define $V_{i j}$ as the traffic on the edge $\left(A_{i}, A_{j}\right)$ which is the actual traffic volume of the road.

Under the premise that roads are not congested, bigger the actual traffic volume of the road is better. Namely find the maximum flow from the origin point $A_{s}$ to the destination $A_{t}$ without exceeding the capacity of each edge.

To compute the maximum flow, define the feasible flow firstly, which satisfies the following conditions:

(1) capacity constraints (The flow on each edge can't exceed the capacity of the edge to prevent congestion)

$$
0 \leq V_{i j} \leq C_{i j}
$$

(2) Equilibrium condition (For the intermediate point, the incoming flow is equal to the outgoing flow) :

For each intermediate point i $(\mathrm{i} \neq \mathrm{s}, \mathrm{t}): \sum_{j:\left(\mathrm{A}_{i}, A_{j}\right) \in E} V_{i j}-\sum_{j:\left(A_{j}, A_{i}\right) \in E} V_{j i}=0$

For the origin point $A_{s}$ :

$$
\sum_{\left(A_{s}, A_{j}\right) \in E} V_{s j}-\sum_{\left(A_{j}, A_{s}\right) \in E} V_{j s}=A(V)
$$

For the destination $A_{t}$ :

$$
\sum_{\left(A_{t}, A_{j}\right) \in E} V_{t j}-\sum_{\left(A_{j}, A_{t}\right) \in E} V_{j t}=-A(V)
$$

Where $A(V)$ is the flow of the feasible flow, namely the output of the origin point. Thus, the maximum flow can be calculated by the linear programming model $\mathrm{A}(\mathrm{Vmax})$.

$$
\begin{aligned}
& \max A(V), \\
& \text { s.t. } \sum_{j:\left(A_{A}: A\right) \in E} V_{i j}-\sum_{j:\left(A_{A ; A}\right) \in E} V_{j i}=\left\{\begin{array}{l}
A(V), i=s \\
-A(f), i=t \\
0, i \neq s, t
\end{array}\right. \\
& 0 \leq V_{i j} \leq C_{i j}, \forall\left(A_{i}, A_{j}\right) \in E
\end{aligned}
$$


According to the above formula, the maximum flow $\mathrm{A}(\mathrm{Vmax})$ can be calculated, which is the maximum traffic flow from the starting point to the receiving point. When $A(V m a x)$ increase, the overall carrying capacity of the road will rise accordingly. If the overall actual traffic volume doesn't change and the overall area of the traffic saturation declines at the same time, the overall road condition will be improved.

As the district opens to increase the number of roads, regional saturation decline is predictable. However, the intra-district road structure is likely to adversely affect the travel time of the vehicle. Therefore, it is necessary to calculate the minimum time consumed by the maximum traffic flow from point $A_{s}$ to point $A_{t}$ before and after the opening to establish the maximum flow minimum time model.

On each side of a given network $D=(A, E)$, in addition to the capacity attribute, the time cost is need to be added. The corresponding time cost is obviously different under different circumstances. The maximum flow minimum time model is to find a maximum flow from the origin point to the destination point, so that the total travel time is the shortest.

$$
\begin{aligned}
& \min T=\sum_{\left(A_{i}, A_{j}\right) \in E} t_{i j} V_{i j}, \\
& \text { S.t. } 0 \leq V_{i j} \leq C_{i j}, \forall\left(A_{i}, A_{j}\right) \in E, \\
& \sum_{j:\left(A_{i}, A_{j}\right) \in E} V_{i j}-\sum_{j:\left(A_{j}, A_{i}\right) \in E} V_{j i}=q_{i}
\end{aligned}
$$

Among them,

$$
q_{i}=\left\{\begin{array}{l}
A\left(V_{\max }\right), i=s \\
-A\left(V_{\max }\right), i=t \\
0, i \neq s, t
\end{array}\right.
$$

After the opening of the community, the number of roads will increase, and the number of edges and vertices will also increase correspondingly, just as the Figure 2.

However, we can't simply consider the number of open areas as the increase in the number of roads. The district will introduce a new intersection after the opening, resulting in a certain delay. Thus the main road speed is affected. The original road intersection will also have a delayed impact on traffic flow, but it can be considered that the impact of the original intersection before and after the opening of the district does not change.

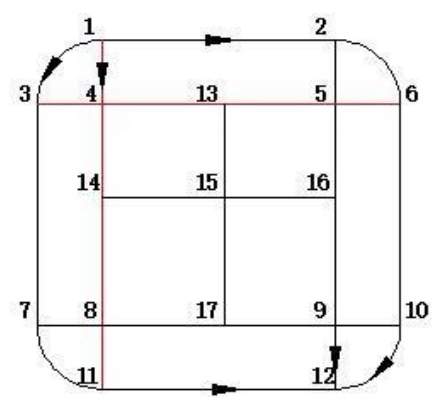

Fig. 2 Community open

Right Turn Delay Correction for Intersection: Traffic lights time and the effect of turning on straight make the car within the district speed limit and also increase the delay time of intersection turning. It can be expressed as ${ }^{[3]}$

$$
d_{r}=K_{0} \frac{1}{60} e^{K_{1} \frac{V}{K_{2} C_{0}(0.6 N+0.4) \chi\left\{1-P_{R T}\left[K_{3}+\left(V_{B B} / K_{4}\right)\right]\right\}}}
$$

In the formula, $\mathrm{V}$ is the path of the actual traffic volume; $C_{0}$ is the maximum amount of traffic connected with the intersection of roads; $\mathrm{N}$ is the intersection of imported motor vehicle lane to the right number; $\chi$ is the capacity reduction coefficient; $f_{R}=1-P_{R T} \times\left[K_{3}+V_{B B} / K_{4}\right]$ is the right to split 
the correction coefficient; $K_{2}$ is the adjustment coefficient; $K_{0} 、 K_{1} 、 K_{3} 、 K_{4}$ are undetermined coefficient.

Left Turn Delay Correction for Intersection: Turning left at the intersection of motor vehicles is more complex than the right turn, and left turn motor vehicles will be affected by straight line of cars. It can be expressed as:

$$
d_{l}=K_{0} \frac{1}{60} e^{K_{1} \frac{V}{K_{2} C_{0}(0.6 N+0.4) \beta f_{L T} f_{L B}}}
$$

The same coefficients are explained in the above.

\section{Verification of the proposed model}

Calculate the above typical case: The maximum flow before opening: $A\left(V_{\max }\right)=189 \mathrm{pcu} / \mathrm{min}$.And the maximum flow after opening: $A\left(V_{\max }\right)=189 \mathrm{pcu} / \mathrm{min}$.It indicates that the community does not increase the maximum flow of traffic. Moreover, the total travel time after opening is $\mathrm{T}=39662.40$, and the average travel time is 209.5, which is larger than before opening. This shows that, after the opening of the district, the maximum flow for the surrounding is not increased but the delay caused by the intersection of the maximum flow has increased.

Select the case II as shown below. $\mathrm{T}=47147.9$, and the average travel time is 142.87 . We find that for this case not only the maximum flow is significantly increased, but also the average travel time at the maximum flow is effectively reduced, indicating that the openness of the area has markedly improved the road network in this area.

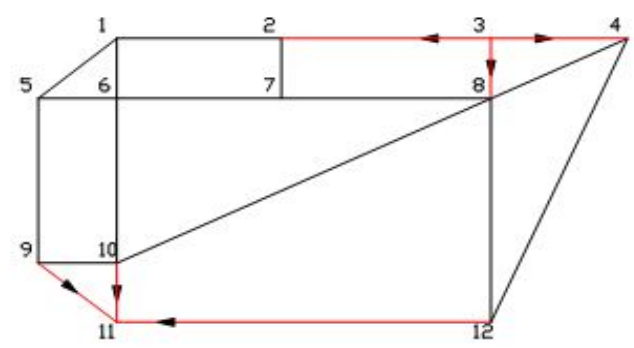

Fig. 3 Another community road map

\section{Conclusion}

Strong community perimeter road and low - grade community internal roads can't play the same as the connection of capillaries. So the opening of the district doesn't increase the maximum flow of this area and makes the average travel time longer.

Weak community perimeter road and urban trunk road on both sides of the district can be the same to the capillaries which strengthen the trunk road. Although it causes some delays in the original road, it complements the lack of peripheral roads. So it can play a role in expanding the maximum flow area and reducing the average travel time at the same time.

\section{References}

[1] BAO Yuan2qiu and YAN Ling, Research and application of the turn penalty function in the transportation planning, J. University of Shanghai for Science and Technology, Vol.27, No.3, pp.253-258,2005

[2] Information on http://wenku.baidu.com/

[3] JIANG Gui2yan, LI Ji2wei and ZHANG Chun2qin, Modified BPR Functions for Travel Time Estimation of Urban Arterial Road Segment, Journal Of Southwest JIAOTONG University, Vol.45 No.1, pp.124-129, Feb. 2010 